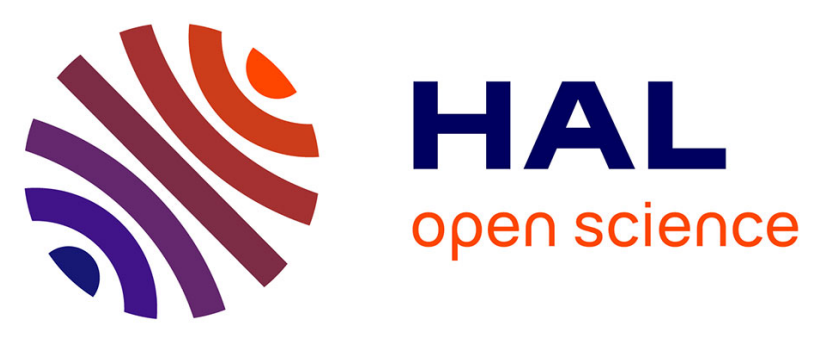

\title{
Effective Elastic Properties Using Maxwell's Approach for Transversely Isotropic Composites
}

\author{
Leandro Daniel Lau Alfonso, Reinaldo Rodríguez-Ramos, José A. Otero, \\ Frédéric Lebon, Federico J Sabina, Raúl Guinovart-Díaz, Julian \\ Bravo-Castillero
}

\section{To cite this version:}

Leandro Daniel Lau Alfonso, Reinaldo Rodríguez-Ramos, José A. Otero, Frédéric Lebon, Federico J Sabina, et al.. Effective Elastic Properties Using Maxwell's Approach for Transversely Isotropic Composites. State of the Art and Future Trends in Material Modeling, pp.183-210, 2019, 10.1007/9783-030-30355-6_9. hal-02336799

\section{HAL Id: hal-02336799 https://hal.science/hal-02336799}

Submitted on 8 Oct 2020

HAL is a multi-disciplinary open access archive for the deposit and dissemination of scientific research documents, whether they are published or not. The documents may come from teaching and research institutions in France or abroad, or from public or private research centers.
L'archive ouverte pluridisciplinaire HAL, est destinée au dépôt et à la diffusion de documents scientifiques de niveau recherche, publiés ou non, émanant des établissements d'enseignement et de recherche français ou étrangers, des laboratoires publics ou privés. 


\title{
Effective Elastic Properties Using Maxwell's Approach for Transversely Isotropic Composites
}

\author{
Leandro Daniel Lau Alfonso, Reinaldo Rodríguez-Ramos, Jose A. Otero, Frédéric \\ Lebon, Federico J. Sabina, Raul Guinovart-Díaz, and Julián Bravo-Castillero
}

\begin{abstract}
In this contribution an analysis of static properties of transversely isotropic, porous and nano-composites is considered. Present work features explicit formulas for effective coefficient in these types of composites. The reinforcements of the

\footnotetext{
Leandro Daniel Lau Alfonso

Instituto de Cibernética, Matemática y Física, ICIMAF, Calle 15 No. 551, entre C y D, Vedado, Habana 4, CP-10400, Cuba,

e-mail: leandro@icimaf.cu

Reinaldo Rodríguez-Ramos

Facultad de Matemática y Computación, Universidad de la Habana, San Lázaro y L, Vedado, Habana 4, CP-10400, Cuba \& Visiting Professor from February 15 to August 15, 2019 at Instituto de Investigaciones en Matemáticas Aplicadas y en Sistemas, Universidad Nacional Autónoma de México, Alcaldía Álvaro Obregón, 01000 CDMF, México,

e-mail: reinaldo@matcom.uh. cu
}

Jose A. Otero

Instituto Tecnológico de Estudios Superiores de Monterrey, CEM, E.M. CP 52926, México,

e-mail: j.a.otero@tec.mx

Frédéric Lebon

Aix-Marseille University, CNRS, Centrale Marseille, LMA, 4 Impasse Nikola Tesla, CS 40006, 13453 Marseille Cedex 13, France,

e-mail: lebon@lma.cnrs-mrs.fr

Federico J. Sabina

Instituto de Investigaciones en Matemáticas Aplicadas y en Sistemas, Universidad Nacional Autónoma de México, Alcaldía Álvaro Obregón, 01000 CDMF, México,

e-mail: fjs@mym. iimas. unam.mx

Raul Guinovart-Díaz

Facultad de Matemática y Computación, Universidad de la Habana, San Lázaro y L, Vedado, Habana 4, CP-10400, Cuba,

e-mail: guino@matcom.uh. cu

Julián Bravo-Castillero

Instituto de Investigaciones en Matemáticas Aplicadas y en Sistemas, Universidad Nacional Autónoma de México, Alcaldía Álvaro Obregón, 01000 CDMF, México,

e-mail: julian@mym. iimas. unam.mx 
composites are a set of spheroidal inclusions with identical size and shape. The center is randomly distributed and the inclusions are embedded in an homogeneous infinite medium (matrix). An study of theoretical predictions obtained by Maxwell approach using two different density distribution functions, which describe the alignment inclusions is done. The method allows to report the static effective elastic coefficients in composites ensemble with inclusions of different geometrical shapes and configurations embedded into a matrix. The effective properties of composites are computed using the Maxwell homogenization method in Matlab software. Another novelty of this contribution is the calculation of new explicit analytical formulas for the control of the alignment tensors $\mathbf{N}^{*}$ and $\mathbf{N}^{s *}$ which is in charged of the alignment distribution of inclusions within matrix through disorder parameters $\lambda$ and $s$, respectively. The alignment tensors $\mathbf{N}^{*}$ and $\mathbf{N}^{s *}$ are obtained as average of all possible alignments of the inclusions inside the composite. Numerical results are obtained and compared with some other theoretical approaches reported in the literature.

Keywords: Maxwell's scheme · Inhomogeneities · Homogenization · Transversely isotropic composites

\subsection{Introduction}

It was the scientist James Clerck Maxwell, who proposed a method to calculate the effective conductivity of a homogeneous spherical material that contained a finite amount of inclusions of spherical type in Maxwell (1954). This aroused the interest of the scientific community since in its method Maxwell did not consider interactions between inclusions and arrived, in the case of spherical inclusions, to the same predictions as other methods that take them into account.

In Kanaun (2016); Levin and Kanaun (2012) recent results are presented in this area. In Kanaun (2016) four methods are compared, the original and generalized Maxwell schemes and the one-particle and multi-particle effective field methods (EFM). Those approaches give closed predictions for small volume fractions of inclusions $(p<0.3)$, furthermore, another method such as (MT) method is mentioned, concluding that in the case of isotropic materials with spherical inclusions of equal behaviour, the original Maxwell and the one-particle effective field methods, coincide in their results, and in the case of spheroidal inclusions the original Maxwell and the one-particle EFM deviate substantially from the multi-particle EFM and generalized Maxwell scheme, being remarkable that both last approaches coincide practically for all the values of the volume fraction. The Maxwell method is extended in Levin and Kanaun (2012) to an homogeneous anisotropic medium containing an arbitrary set of homogeneous anisotropic ellipsoidal inclusions, where it is shown that the explicit equations obtained by the Maxwell method for isotropic materials coincide in the case of spherical inclusions with the MT, and for spheroidal inclusions oriented in a 
parallel direction, the equations for the effective elastic modulus tensor are the same, which are obtained by Maxwell and MT methods.

In addition, in Sevostianov (2014), Maxwell's scheme is reformulated from the rigidity and flexibility contribution tensors, being remarkable that the form of the fictitious building, affects the predictions of the effective coefficients. Moreover, explicit formula for choosing the aspect ratio of fictitious building is given when it presents spheroidal shape, and the advantages of the Maxwell method are proposed. It shows that the reformulation of its scheme as a function of the tensor of contribution of flexibility, is equivalent to the reformulation as a function of the tensor of contribution of rigidity. In Sevostianov and Giraud (2013), the Maxwell method reformulated as a function of the rigidity contribution tensor is illustrated, through four examples: the first is a material that contains multiple pores of identical form, the second, a material that contains three families of inclusions that have different properties and forms, the third, a material that contains circular cracks with certain orientations and, finally, a material containing randomly oriented pores with no ellipsoidal shapes.

In Martinez-Ayuso et al (2017) a study is presented on the homogenization of piezoelectric materials with pores, through numerical and analytical methods. The results obtained in Martinez-Ayuso et al (2017) are compared by two different methods: (MT) method and self-consistent scheme of Hershey (1954) and Kröner (1958). Besides, these results are contrasted with two classical bounds known in the homogenization theory, the Hashin-Strikman and Halpin-Tsai bounds. In addition, a numerical model (FEM) of representative volume element is developed, based on the analysis of finite element for different percentage of inclusions in the material. The locally exact homogenization theory for unidirectional composites with square periodicity and isotropic phases proposed by Drago and Pindera (2008) is extended in Wang and Pindera (2016) to architectures with hexagonal symmetry and transversely isotopic phases, through a numerical method that uses Fourier transformation.

In McCartney and Kelly (2008) the far-field methodology developed by Maxwell (1954), is used to estimate effective thermoelastic properties in multi-phase isotropic composites. Furthermore, effective bulk and shear moduli are estimated, as well as thermal expansion coefficients in these types of composites. Besides, results are compared with formulas and dimensions, known in the literature. The generalized Maxwell method is developed by Levin et al (2012) for the calculation of effective parameters in poroelastic composites. This method is compared with other selfconsistent methods existing in the literature. Moreover, examples of applications of the generalized method of Maxwell for the calculation of effective parametric parameters for heterogeneous materials constituted by rocks are reported. The Maxwell homogenization scheme (Kushch and Sevostianov, 2016) is formulated in terms of moments of dipole and of the tensor of contribution of element of representative volume, also deals with the problem of effective conductivity in a composite with spheroidal inclusions aligned, analyzing the convergence of that solution.

The novelty of the present contribution is the derivation of explicit analytical formulae for the control of the alignment tensors $\mathbf{N}^{*}$ and $\mathbf{N}^{s *}$. These functions distribute the alignment of inclusions inside the matrix material through disorder parameters $\lambda$ and $s$, respectively, obtained as an average of all possible alignments of 
the inclusions within the nano transversely isotropic composite. Another novelty of this contribution consists in the study of Maxwell's approach predictions using two different density distribution functions (Sevostianov, 2014; Giraud et al, 2007) for alignment inclusions inside the composite. The different types of inclusions taken into account in the model are spheroidals. Spheroidal fibre, spherical inclusion and spheroidal disk are considered for different ranges of the aspect ratio parameter. Moreover, explicit formulae for effective elastic tensor are given for porous, nano and composites with global transversely isotropic behaviour formed by constituents with transversely isotropic symmetry as well. Comparisons with other theoretical approaches, such as, closed relations reported by Christensen model and FEM (Dong et al, 2005), Locally-exact homogenization theory (LEHT) (Wang and Pindera, 2016), among others are given.

\subsection{Statement of Fundamental Equations}

A solid material of volume $V$ that posses linear elastic behaviour is considered. In this case, the constitutive equations for a linear elastic solid can be written in terms of stress tensor $\sigma_{i j}$ and strain tensor $\varepsilon_{i j}$ through Hooke law

$$
\sigma_{i j}=C_{i j k l} \varepsilon_{k l}
$$

where $C_{i j k l}$ is the stiffness fourth order tensor. In previous equation, the indexes $i, j, k, l$ go from 1 to 3 . The elastic constants satisfy the following symmetry relationships

$$
C_{i j k l}=C_{j i k l}=C_{i j l k}
$$

The symmetry of elastic constants (9.2) reduces the number of elastic independent constants from 81 to 36 (see Qu and Cherkaoui, 2006; Roger and Dieulesaint, 2000). For crystals, stiffness tensor $C_{i j k l}$, formed by 36 components, is also symmetric respect to permutation of pairs of indexes

$$
C_{i j k l}=C_{k l i j}
$$

The existence of equality (9.3) in the general case, lead to a reduction of the number of independent components for the stiffness tensor from 36 to 21, that is, the number of constants of a solid without symmetry.

The strain $\boldsymbol{\varepsilon}$ and displacement $\boldsymbol{u}$ are related by the Cauchy linear relationship

$$
\varepsilon_{i j}=\frac{1}{2}\left(u_{i, j}+u_{j, i}\right)
$$

The components of elastic tensor moduli, strain and stress in matrix notation, is useful to write using the abbreviate notation. The binary combinations $i j=m(i, j=1,2,3)$ and $k l=n(k, l=1,2,3)$ are substituted by an index from 1 to 6 following the next scheme $(m, n=1, \ldots, 6)$ 


$$
(11) \rightarrow 1 ;(22) \rightarrow 2 ;(33) \rightarrow 3 ;(23,32) \rightarrow 4 ;(31,13) \rightarrow 5 ;(12,21) \rightarrow 6 .
$$

Equation (9.1) can be written in matrix notation

$$
\left(\begin{array}{l}
\sigma_{1} \\
\sigma_{2} \\
\sigma_{3} \\
\sigma_{4} \\
\sigma_{5} \\
\sigma_{6}
\end{array}\right)=\left(\begin{array}{llllll}
C_{11} & C_{12} & C_{13} & C_{14} & C_{15} & C_{16} \\
C_{21} & C_{22} & C_{23} & C_{24} & C_{25} & C_{26} \\
C_{31} & C_{32} & C_{33} & C_{34} & C_{35} & C_{36} \\
C_{41} & C_{42} & C_{43} & C_{44} & C_{45} & C_{46} \\
C_{51} & C_{52} & C_{53} & C_{54} & C_{55} & C_{56} \\
C_{61} & C_{62} & C_{63} & C_{64} & C_{65} & C_{66}
\end{array}\right)\left(\begin{array}{l}
\varepsilon_{1} \\
\varepsilon_{2} \\
\varepsilon_{3} \\
\varepsilon_{4} \\
\varepsilon_{5} \\
\varepsilon_{6}
\end{array}\right) .
$$

Material's behavior is described through 21 constants of the tensor $C_{i j k l}$.

The elastic fourth rank stiffness tensor for composites with transversely isotropic symmetry, oriented along the $x_{3}$ symmetry axis, is given by

$$
\mathbf{C}=\left(\begin{array}{cccccc}
C_{11} & C_{12} & C_{13} & 0 & 0 & 0 \\
C_{12} & C_{11} & C_{13} & 0 & 0 & 0 \\
C_{13} & C_{13} & C_{33} & 0 & 0 & 0 \\
0 & 0 & 0 & C_{44} & 0 & 0 \\
0 & 0 & 0 & 0 & C_{44} & 0 \\
0 & 0 & 0 & 0 & 0 & \frac{C_{11}-C_{12}}{2}
\end{array}\right)
$$

As a particular case, if the material has isotropic symmetry, the independent constants are reduced to 2 . Thus, constitutive equation (9.6) can be written

$$
\left(\begin{array}{l}
\sigma_{1} \\
\sigma_{2} \\
\sigma_{3} \\
\sigma_{4} \\
\sigma_{5} \\
\sigma_{6}
\end{array}\right)=\left(\begin{array}{cccccc}
C_{11} & C_{12} & C_{12} & 0 & 0 & 0 \\
C_{12} & C_{11} & C_{12} & 0 & 0 & 0 \\
C_{12} & C_{12} & C_{11} & 0 & 0 & 0 \\
0 & 0 & 0 & \frac{1}{2}\left(C_{11}-C_{12}\right) & 0 & 0 \\
0 & 0 & 0 & 0 & \frac{1}{2}\left(C_{11}-C_{12}\right) & 0 \\
0 & 0 & 0 & 0 & 0 & \frac{1}{2}\left(C_{11}-C_{12}\right)
\end{array}\right)\left(\begin{array}{c}
\varepsilon_{1} \\
\varepsilon_{2} \\
\varepsilon_{3} \\
\varepsilon_{4} \\
\varepsilon_{5} \\
\varepsilon_{6}
\end{array}\right) .
$$

In some cases, it is convenient to represent stiffness tensor $C_{i j k l}$ for isotropic materials in the form

$$
C_{i j k l}=\lambda \delta_{i j} \delta_{k l}+\mu\left(\delta_{i k} \delta_{j l}+\delta_{i l} \delta_{j k}\right)=K \delta_{i j} \delta_{k l}+G\left(\delta_{i k} \delta_{j l}+\delta_{i l} \delta_{j k}-\frac{2}{3} \delta_{i j} \delta_{k l}\right),
$$

where $K$ and $G$ are the bulk and shear modulus, respectively, and $\lambda$ and $\mu$ are Lame's constants, which are related with the constants of tensor $C_{i j k l}$ of Eq. (9.8) by

$$
\begin{aligned}
& C_{11}=C_{22}=C_{33}=\lambda+2 \mu, \\
& C_{12}=C_{13}=C_{23}=\lambda, \\
& C_{44}=C_{55}=C_{66}=\mu=\left(C_{11}-C_{12}\right) / 2 .
\end{aligned}
$$


The stress field given by stress tensor represents an equilibrium state with volume forces $f_{i}$ in all the points of the volume $V$, it means that stress field satisfies the equilibrium equation

$$
\frac{\partial \sigma_{i j}}{\partial x_{j}}+f_{i}=0 .
$$

Equation (9.11) is valid for any material point inside a continuum medium.

Introducing $\mathbf{T}$ basis (Sevostianov, 2014) for transversely isotropic tensor

$$
\begin{gathered}
T_{i j k l}^{1}=\Theta_{i j} \Theta_{k l}, \quad T_{i j k l}^{2}=\frac{\Theta_{i k} \Theta_{l j}+\Theta_{i l} \Theta_{k j}-\Theta_{i j} \Theta_{k l}}{2}, \quad T_{i j k l}^{3}=\Theta_{i j} \xi_{k} \xi_{l}, \\
T_{i j k l}^{4}=\xi_{i} \xi_{j} \Theta_{k l}, \quad T_{i j k l}^{5}=\frac{\Theta_{i k} \xi_{l} \xi_{j}+\Theta_{i l} \xi_{k} \xi_{j}+\Theta_{j k} \xi_{l} \xi_{i}+\Theta_{j l} \xi_{k} \xi_{i}}{4}, \\
T_{i j k l}^{6}=\xi_{i} \xi_{j} \xi_{k} \xi_{l}, \quad \Theta_{i j}=\delta_{i j}-\xi_{i} \xi_{j}, \\
\xi=\left(\xi_{1}, \xi_{2}, \xi_{3}\right)=(\sin \psi \cos \theta, \sin \psi \sin \theta, \cos \psi), \quad \psi \in[0, \pi], \theta \in[0,2 \pi],
\end{gathered}
$$

where $\delta_{i j}$ is the Kronecker delta, and using decomposition of stiffness tensor (9.7) in T basis oriented along $x_{3}$ symmetry axis (Sevostianov, 2014), it holds

$$
\begin{aligned}
& \mathbf{C}=k \mathbf{T}^{1}+2 m \mathbf{T}^{2}+l\left(\mathbf{T}^{3}+\mathbf{T}^{4}\right)+4 \mu \mathbf{T}^{5}+n \mathbf{T}^{6}, \\
& \mathbf{C}=(k, 2 m, l, l, 4 \mu, n),
\end{aligned}
$$

where

$$
\begin{aligned}
& k=\frac{C_{1111}+C_{1122}}{2}, \quad m=\frac{C_{1111}-C_{1122}}{2}, \\
& l=C_{1133}, \quad \mu=C_{2323}, \quad n=C_{3333},
\end{aligned}
$$

where $k$ is the plane-strain bulk modulus for lateral dilatation without longitudinal extension, $m$ is the rigidity modulus for shearing in any transverse direction, $l$ is the associated cross-modulus, $\mu$ is the longitudinal or axial shear modulus, and $n$ is the modulus for longitudinal uniaxial strain.

\subsection{Geometry of Inclusions}

Initially a composite material is considered on the framework of Maxwell approach, with a fictitious building (the fictitious building is an arbitrary region that is framed within the composite) of spheroidal shape inside the homogeneous material (matrix). The composite can be ensemble, in principle, for a single type of inclusion or different types of spheroidal inclusions. In Fig. 9.1 it is featured a sample of the composite with the fictitious building (region in dark gray) inside matrix material (region in normal gray). Three different types of spheroidal inclusions accounted in the present model are described in a mathematical form through the set 
Fig. 9.1 Representative nano transversal isotropic material with spheroidal inclusions and fictitious building

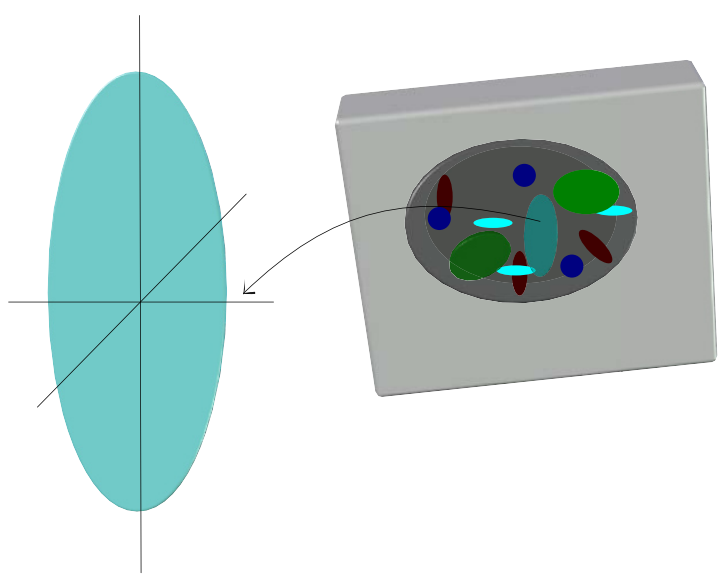

$$
\mathfrak{I}=\left\{(x, y, z) \in \mathbb{R}^{3}: \frac{x^{2}}{(\gamma a)^{2}}+\frac{y^{2}}{(\gamma a)^{2}}+\frac{z^{2}}{a^{2}} \leq 1, a \in \mathbb{R}\right\},
$$

where the parameter $\gamma$, is denominated aspect ratio of the inclusions. In the present model, the parameter $\gamma$ is taken as $\gamma=\frac{x_{1}}{x_{3}}$, taking the value $\gamma=1$ for spherical inclusions, $\gamma<1$ for fibre cylindrical inclusions and $\gamma>1$ for spheroidal disk inclusions, Fig. 9.2. Analogously, the aspect ratio of the fictitious building within matrix material is assigned to the parameter $\Gamma$ and it describes the geometrical form of this construction inside the composite through the set

$$
F_{B}=\left\{(x, y, z) \in \mathbb{R}^{3}: \frac{x^{2}}{(\Gamma b)^{2}}+\frac{y^{2}}{(\Gamma b)^{2}}+\frac{z^{2}}{b^{2}} \leq 1, b \in \mathbb{R}\right\} .
$$

The different types of inclusions taken into account in the model are spheroidals. In Fig. 9.1 all possible spheroidal inclusions embedded into the fictitious body is featured and in Fig. 9.2 spheroidal fibre, spherical and spheroidal disk inclusions are shown for different ranges of the aspect ratio $\gamma$.

Fig. 9.2 Description of three different types of spheroidal inclusions taking into account in the present model
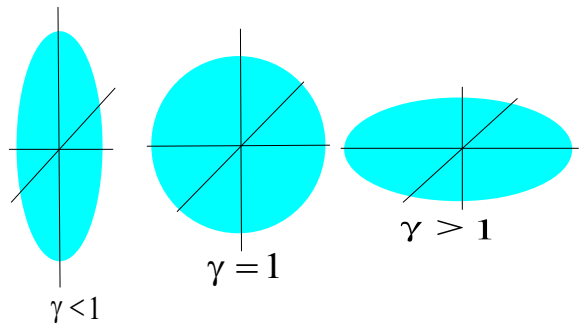


\subsection{Maxwell's Homogenization Approach}

Let the effective stiffness tensor $\mathbf{C}^{*}$ of a transversely isotropic elastic composite, with different types of spheroidal inclusions $\Omega_{m}, m=1, \ldots, n$ of volume $V_{m}$, and be $\mathbf{C}^{0}$ and $\mathbf{C}^{i}$ the matrix and $i$ th-inclusion stiffness tensor, respectively, being $V$ the volume of the whole composite. Then, by Maxwell approach, a fictitious building $\Omega$ of volume $\bar{V}$ is taken inside the composite formed by matrix material and inclusions, the resulting effect due to applying a constant external strain field $\varepsilon^{0}$ (this fact is shown in Fig. 9.3) to the matrix material is described by the sum of stiffness contribution tensor of inclusions $\frac{1}{V} \sum_{i} V_{i} \mathbf{N}_{i}$ and by the stiffness contribution tensor $\overline{\mathbf{N}}$ (Sevostianov, 2014) of $\Omega$, considering $\Omega$ as an individual inclusion with homogeneous unknown properties $\mathbf{C}^{*}$, which volume is representative in the whole composite. Later, equating both produced fields (in a further point $\rho$ of the composite), by inclusions and $\Omega$, considering this last one like independent inclusion, it holds the effective equation obtained by Maxwell approach

$$
\frac{\bar{V}}{V} \overline{\mathbf{N}}=\frac{1}{V} \sum_{i} V_{i} \mathbf{N}_{i}
$$

Given in the formula (9.36)-(9.38) of the Appendix, tensor $\mathbf{N}$ in the Maxwell approach contains the information about the geometrical shape and elastic properties of the inclusions, it also depends on the elastic properties of the matrix material through the components of tensor $\mathbf{P}$ which contains the aspect ratio $\gamma$ in its integral expressions (9.27). The tensor $\mathbf{P}$ (Hill, 1965) describes the geometrical shape and properties of fictitious building and it contains the elastic properties of matrix material and the aspect ratio of the fictitious building $\Gamma$, and for ellipsoidal inclusions the following relation holds

Fig. 9.3 Featuring of fictitious building in matrix material

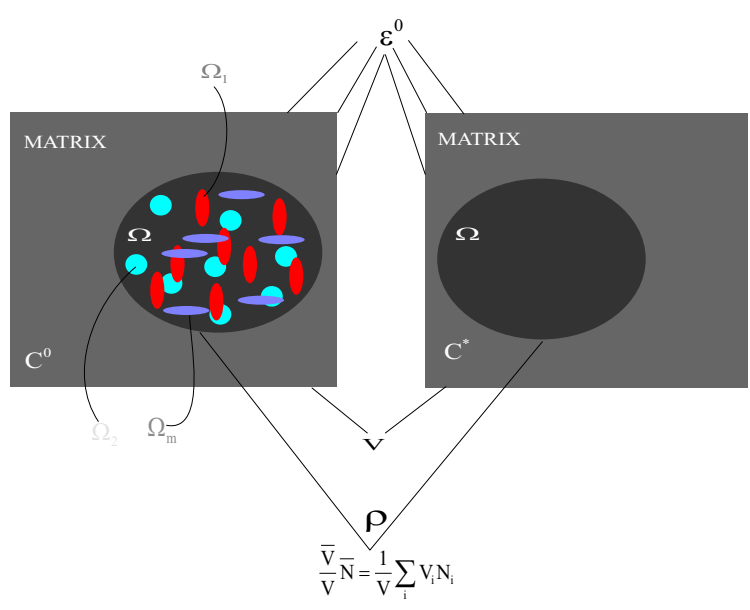




$$
\mathbf{N}=\left[\left(\mathbf{C}^{i}-\mathbf{C}^{0}\right)^{-1}+\mathbf{P}\right]^{-1}
$$

and tensor $\mathbf{P}$ is calculated

$$
\begin{aligned}
\mathbf{P}_{i j k l} & =\int_{\Psi} K_{i j k l}^{*}\left(a^{-1} k\right) d \Psi, \quad K_{i j k l}(x)=-\left[\nabla_{j} \nabla_{l} G_{i k}(x)\right]_{(i j)(k l)}, \\
\mathbf{a} & =\left(\begin{array}{ccc}
1 / a_{1} & 0 & 0 \\
0 & 1 / a_{2} & 0 \\
0 & 0 & 1 / a_{3}
\end{array}\right),
\end{aligned}
$$

where $\Psi$ is the unit sphere, $a_{1}, a_{2}, a_{3}$ are the axis of the ellipsoidal volume $V, G_{i k}$ is the static Green function of operator $\nabla_{j} C_{i j k l}^{0} \nabla_{l}$ and $K_{i j k l}^{*}$ the Fourier transform of function $K_{i j k l}$, given by

$$
K_{i j k l}^{*}=\frac{1}{4}\left[\xi_{j}\left(\mathbf{G}_{i k}\right)^{-1} \xi_{l}+\xi_{i}\left(\mathbf{G}_{j k}\right)^{-1} \xi_{l}+\xi_{j}\left(\mathbf{G}_{i l}\right)^{-1} \xi_{k}+\xi_{i}\left(\mathbf{G}_{j l}\right)^{-1} \xi_{k}\right],
$$

where $\xi_{j}$ are given by (9.15). From (9.19) it holds that when $\Omega$ has ellipsoidal form

$$
\overline{\mathbf{N}}=\left[\left(\mathbf{C}^{*}-\mathbf{C}^{0}\right)^{-1}+\mathbf{P}\right]^{-1}
$$

and then by substitution (9.23) into (9.18), it yields the final expression for effective stiffness tensor of composite

$$
\mathbf{C}^{*}=\mathbf{C}^{0}+\left[\left(\frac{1}{\bar{V}} \sum_{i} V_{i} \mathbf{N}_{i}\right)^{-1}-\mathbf{P}\right]^{-1}
$$

Equation (9.24) is the most important formula of Maxwell method because it allows to write the explicit expression for computing effective stiffness tensor of composites. Then, following the idea of (Sevostianov, 2014), one can replace in eq. (9.24) the sum $\sum_{i} \mathbf{N}_{i}$ by the quantity $\mathbf{N}^{*}$ given by formulae (9.49)-(9.54) of the Appendix, and the quantity $\sum_{i} \frac{V_{i}}{\bar{V}}$ by parameter $v_{f}$ which denotes the volume fraction of inclusion in the model, consequently, for two-phase composites Eq. (9.24) becomes

$$
\mathbf{C}^{*}=\mathbf{C}^{0}+v_{f} \cdot\left[\left(\mathbf{N}^{*}\right)^{-1}-v_{f} \cdot \mathbf{P}\right]^{-1},
$$

Moreover, the Maxwell approach allows us to replace tensor $\mathbf{N}^{*}$ of Eq. (9.25) by tensor $\mathbf{N}^{s *}$ given by formulae (9.65)-(9.76) of the Appendix for obtaining two Maxwell homogenization approaches that differ one from the other in the density distribution function chosen for modeling the alignment of inclusions within matrix materials. 


\subsection{Analysis of Numerical Results}

In this section, present model (PM) using the Maxwell method applied to nanocomposites and transversely isotropic composite materials is validated with other theoretical approaches reported in different works. In Table 9.1 are shown the material properties used in the calculations.

The present model is compared with Christensen, FEM approach (Table 1 of Dong et al (2005)) and Locally Exact Homogenization Theory (Wang and Pindera, 2016) for validating the case of composite with isotropic matrix and transversely isotropic inclusion. Besides, a comparison with Mogilevskaya et al (2014) for effective tetragonal elastic moduli of two phase fiber reinforced composite is done. Furthermore, a validation for the porous inclusion case with respect to Sevostianov (2014); Vilchevskaya and Sevostianov (2015) approaches is performed. The present model (PM) is compared with the approach reported in Selmi et al (2007) for the case of two phase nanocomposite. Moreover, a study about the influence on the effective coefficient through PM using two different density distribution functions (DDF) for describing parallel alignment inclusions within matrix is reported. From now on, DDF given by (9.47) and (9.63) are identified by DDF1 and DDF2, respectively. The procedure given by the Eq. (9.25) using tensor $\mathbf{N}^{*}$ reported in (9.48) and $\mathbf{N}^{s *}$ given in (9.64) is denoted by PM-DDF1 and PM-DDF2, respectively. The numerical results shown in the figures are taken from the mentioned literature in each study. Present model's predictions are based in the effective equation (9.25) obtained in the last section.

Table 9.1 Mechanical properties of the constituents used for the computation

\begin{tabular}{llllll}
\hline material properties (GPa) & $C_{1111}$ & $C_{1122}$ & $C_{1133}$ & $C_{3333}$ & $C_{2323}$ \\
\hline Epoxy & 6.64444 & 3.57778 & 3.57778 & 6.64444 & 1.53333 \\
Boron inclusions & 459.667 & 114.917 & 114.917 & 459.667 & 172.375 \\
3501-6 Epoxy & 6.464 & 3.33 & 3.33 & 6.464 & 1.576 \\
AS4 Graphite & 15.6879 & 3.18792 & 3.77517 & 226.51 & 15 \\
matrix & 1 & 0.5 & 0.5 & 1 & 0.25 \\
moderate inclusion & 18 & 15 & 15 & 18 & 1.5 \\
high inclusion & 1200 & 1000 & 1000 & 1200 & 100 \\
matrix1 & 130.27 & 56.0962 & 56.0962 & 130.27 & 37.0869 \\
matrix2 & 17.9482 & 10.1082 & 10.6614 & 32.2627 & 8.75362 \\
porous & $10.1^{-7}$ & $10.12^{-7}$ & $10.13^{-7}$ & $10.14^{-7}$ & $10.15^{-7}$ \\
LaRC-SI & 8.14286 & 5.42857 & 5.42857 & 8.14286 & 1.35714 \\
continuum Graphene & 3024 & 1008 & 1008 & 3024 & 1008 \\
Aluminum & 101.9 & 50.022 & 50.022 & 101.9 & 25.94 \\
SiC & 474.2 & 98 & 98 & 474.2 & 188.1 \\
\hline
\end{tabular}




\subsubsection{Density Distribution Functions}

The influence on effective properties obtained through Maxwell approach using density distribution function given by (9.47) in Appendix denoted by DDF1 for modeling different inclusion alignment cases inside matrix material, is studied by Sevostianov (2014) for porous composite. Now, the purpose is to show the results of Maxwell approach using DDF2 given by (9.63) in Appendix (PM-DDF2) and (PMDDF1) for modeling inclusion's alignment inside composite, and compare these two approaches between them and other predictions reported in other research works not only for porous composites but for a more general variety of different composites like Epoxy/Boron, 3501-6 Epoxy/AS4 Graphite, matrix/moderate inclusion, matrix/high inclusion, among others.

In Fig. 9.4 is shown the dependence on the alignment parameter $s$ of six alignment functions $\tau_{i}$ given by formulae (9.71)-(9.76) of the Appendix when disorder parameter $s \rightarrow \infty, s$ controls the alignment of inclusions inside the matrix material through tensor $\mathbf{N}^{s *}$. Present model takes into account the random or non random alignment of every type of inclusion inside the matrix through parameter $s$. Figure 9.4 reflects that functions $\tau_{i}(s)$ have horizontal asymptotes at different values as $s \rightarrow \infty$. It is remarkable that these six functions remain constants as the disorder parameter $s$ approaches to infinity.

In Table 9.2 are shown the predictions of the tensor $\mathbf{N}^{*}$ numerical values (in $\mathrm{GPa}$ ) given by formulae (9.49)-(9.54) for different values of disorder parameter $\lambda$, for modeling cylindrical inclusions within the matrix in a Epoxy/Boron composite (see Table 9.1), taking into account aspect ratios of inclusions and fictitious building $\gamma=\Gamma=0.01$. From Table 9.2 one can observe that for values of disorder parameter $\lambda$ higher than 100, the components of tensor $\mathbf{N}^{*}$ remain close one each other, it implies that the components of effective elastic tensor are also closed through PM-DDF1 approach.
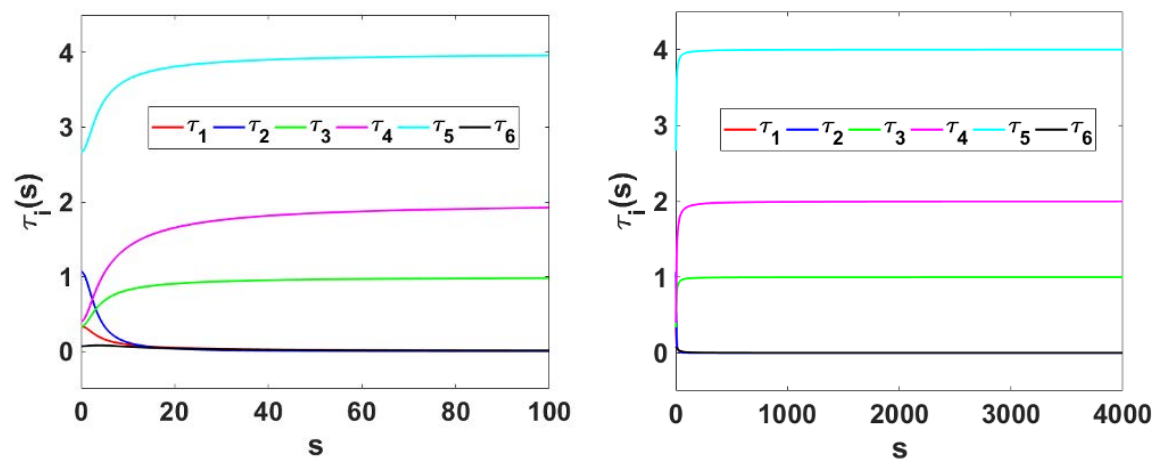

Fig. 9.4 Dependence of alignment functions $\tau_{i}$ given by Eq. (9.71)-(9.76) of the Appendix related to disorder parameter $s$ 
Table 9.2 Numerical predictions for components of tensor $\mathbf{N}^{*}$ which describes the alignment of inclusions inside the composite Epoxy/Boron, $\gamma=\Gamma=0.01$ for fibre inclusions

\begin{tabular}{lllllll}
\hline$\lambda$ & $\frac{\mathbf{N}_{1111}^{*}+\mathbf{N}_{1122}^{*}}{2}$ & $\mathbf{N}_{1111}^{*}-\mathbf{N}_{1122}^{*}$ & $\mathbf{N}_{1133}^{*}$ & $\mathbf{N}_{3311}^{*}$ & $4 \mathbf{N}_{2323}^{*}$ & $\mathbf{N}_{3333}^{*}$ \\
\hline 2 & 33.3135 & 32.5450 & 29.7264 & 29.7264 & 118.2359 & 156.5058 \\
10 & 7.2516 & 5.7731 & 11.2025 & 11.2025 & 45.5601 & 334.8491 \\
100 & 6.4908 & 4.9134 & 2.8862 & 2.8862 & 12.4927 & 371.1578 \\
1000 & 6.4911 & 4.9126 & 2.7783 & 2.7783 & 12.0634 & 371.5879 \\
3700 & 6.4911 & 4.9126 & 2.7773 & 2.7773 & 12.0594 & 371.5920 \\
5000 & 6.4911 & 4.9126 & 2.7773 & 2.7773 & 12.0593 & 371.5921 \\
\hline
\end{tabular}

Results are given in $\mathrm{GPa}$.

In Table 9.3 are shown numerical values (in $\mathrm{GPa}$ ) of tensor $\mathbf{N}^{s *}$ given by formulae (9.65)-(9.70) of Appendix, for different values of disorder parameter $s$, taking into account $\gamma=\Gamma=0.01$ for modeling cylindrical Boron inclusions within epoxy matrix (see the material properties in Table 9.1). From Table 9.3 one can observe that for values of disorder parameter $s$ higher than 3000 , the components of tensor $\mathbf{N}^{s *}$ remain close one each other, it implies that the components of effective elastic tensor are also closed through PM-DDF2 approach. From Fig.9.4 and Tables 9.2 and 9.3 one can conclude that best value of parameter $s$ in PM-DDF2 for estimating PM-DDF1 approach with $\lambda=100$ (aligned inclusion case reported by Sevostianov (2014)) is the value $s=3700$.

\subsubsection{Study of Composites Constituted by Isotropic Matrix and Isotropic Inhomogeneities}

In Table 9.4 it is shown comparisons between present model through two different density distribution functions (DDF) and the results obtained by Christensen, FEM reported in Dong et al (2005) for effective axial bulk $K_{12}^{*}$ and Poisson ratio $v_{31}^{*}$ in a two

Table 9.3 Numerical predictions for components of tensor $\mathbf{N}^{s *}$ which describes the alignment of inclusions within the composite Epoxy/Boron, $\gamma=\Gamma=0.01$ for fibre inclusions

\begin{tabular}{lllllll}
\hline$s$ & $\frac{\mathbf{N}_{1111}^{s *}+\mathbf{N}_{1122}^{s *}}{2}$ & $\mathbf{N}_{1111}^{s *}-\mathbf{N}_{1122}^{s *}$ & $\mathbf{N}_{1133}^{s *}$ & $\mathbf{N}_{3311}^{s *}$ & $4 \mathbf{N}_{2323}^{s *}$ & $\mathbf{N}_{3333}^{s *}$ \\
\hline 2 & 41.3244 & 40.7217 & 30.2001 & 30.2001 & 119.7988 & 122.5679 \\
10 & 11.6640 & 10.4124 & 24.9563 & 24.9563 & 100.1217 & 262.1844 \\
100 & 6.5477 & 5.0052 & 6.2418 & 6.2418 & 25.8454 & 357.5077 \\
1000 & 6.4904 & 4.9156 & 3.1396 & 3.1396 & 13.5013 & 370.1455 \\
3700 & 6.4907 & 4.9133 & 2.8755 & 2.8755 & 12.4503 & 371.2004 \\
5000 & 6.4908 & 4.9131 & 2.8500 & 2.8500 & 12.3487 & 371.3022 \\
\hline
\end{tabular}

Results are given in GPa. 
Table 9.4 Comparisons with Table 1 of Dong et al (2005), $\gamma=\Gamma=0.001$ for fibre inclusions

\begin{tabular}{lllllll}
\hline Effective properties & $v_{f}$ & 0.1 & 0.3 & 0.5 & 0.7 & 0.9 \\
\hline$K_{12}^{*}($ Christensen) & 5.83056 & 7.86606 & 11.4567 & 19.4865 & 53.5138 \\
$K_{12}^{*}($ FEM) & 5.83056 & 7.8661 & 11.457 & 19.487 & 53.514 \\
$K_{12}^{*}($ PM-DDF1) & 5.8305 & 7.8658 & 11.4559 & 19.4835 & 53.4872 \\
$K_{12}^{*}$ (PM-DDF2) & 5.8305 & 7.8658 & 11.4559 & 19.4834 & 53.4868 \\
$v_{31}^{*}($ Christensen) & 0.331156 & 0.296514 & 0.265414 & 0.237339 & 0.211869 \\
$v_{31}^{*}$ (FEM) & 0.33116 & 0.29651 & 0.26541 & 0.23734 & 0.21187 \\
$v_{31}^{*}$ (PM-DDF1) & 0.3323 & 0.2998 & 0.2707 & 0.2443 & 0.2203 \\
$v_{31}^{*}$ (PM-DDF2) & 0.3322 & 0.2995 & 0.2701 & 0.2436 & 0.2195 \\
\hline
\end{tabular}

Results are given in GPa.

phase composite formed by Boron inclusions and Epoxy matrix whose properties are shown in Table 9.1. Both phases, matrix and inclusions exhibit isotropic symmetry. The effective coefficients $K_{12}^{*}, v_{31}^{*}$ are given by formulae (10) of Dong et al (2005). In the calculations are using for both type of DDF, Boron inclusions aspect ratio $\gamma=0.001$, the aspect ratio of fictitious building $\Gamma=0.001$, which is the same to the inclusions. The disorder parameter $\lambda=100$ for modeling parallel aligned inclusion case using the PM-DDF1 approach, and disorder parameter $s=3700$ for PM-DDF2. From Table 9.4 is remarkable that predictions through present model give good agreement with those obtained by Christensen, FEM approaches published in Dong et al (2005).

A comparison with Mogilevskaya et al (2014) and semi-analytical finite element method (SAFEM) reported in Otero et al $(2013,2017)$ implemented for this type of composite under perfect contact is given in Tables 9.5 and 9.6 for tetragonal elastic moduli of two phase fibre reinforced composites. Numerical predictions for the effective coefficient $C_{1313}^{*}$ normalized by the shear modulus $\mu_{0}$ of matrix material for two different composites made of matrix/moderate inclusion and matrix/high inclusion, are shown in Tables 9.5 and 9.6, respectively. All the constituents in both composites are in Table 9.1 and have isotropic symmetry. This comparison corresponds to numerical results obtained by the present approach and different predictions obtained by the expressions (6), (20) and RUC (Repetitive square unit cell) model reported in Mogilevskaya et al (2014). The computations are done for $\gamma=\Gamma=0.001, \lambda=100$ and parameter $s=3700$ for PM-DDF1 and PM-DDF2 approaches, respectively. Table 9.5 shows good agreement between PM-DDF1, PM-DDF2, Eqs. (6), (20) and RUC (Mogilevskaya et al, 2014) as well as SAFEM approaches for matrix/moderate inclusion composite. On the other hand, Table 9.6 reveals good comparison between PM-DDF1, PM-DDF2, RUC and SAFEM approaches, however, it is not the same with the results reported by Eqs. (6) and (20) of Mogilevskaya et al (2014) for the case of matrix/high inclusion composite.

Additionaly, the composites matrix/moderate inclusion and matrix/high inclusions are considered and numerical calculations for the effective coefficient $\left(C_{1111}^{*}-C_{1122}^{*}\right) / 2$ normalized by the shear modulus of matrix $\mu_{0}$ and the effective coefficient $C_{1133}^{*}$ 
Table 9.5 Comparison of the effective coefficient $C_{1313}^{*}$ normalized by the shear modulus of matrix $\mu_{0}$ with Table 5 of Mogilevskaya et al (2014), $\gamma=\Gamma=0.001$ for fibre inclusions in the composite matrix/moderate inclusion

\begin{tabular}{lllllll}
\hline$v_{f}$ & Eq. (6) & Eq. (20) & RUC & PM-DDF1 & PM-DDF2 & SAFEM \\
\hline 0.05 & 1.074 & 1.075 & 1.074 & 1.0742 & 1.0742 & 1.0741 \\
0.1 & 1.154 & 1.155 & 1.154 & 1.1542 & 1.1542 & 1.1538 \\
0.15 & 1.240 & 1.242 & 1.240 & 1.2406 & 1.2405 & 1.2400 \\
0.2 & 1.333 & 1.335 & 1.333 & 1.3341 & 1.3341 & 1.3334 \\
0.25 & 1.435 & 1.437 & 1.435 & 1.4359 & 1.4358 & 1.4351 \\
0.3 & 1.545 & 1.548 & 1.546 & 1.5469 & 1.5468 & 1.5463 \\
0.35 & 1.667 & 1.670 & 1.669 & 1.6685 & 1.6683 & 1.6688 \\
0.4 & 1.800 & 1.804 & 1.805 & 1.8023 & 1.8021 & 1.8045 \\
0.45 & 1.947 & 1.952 & 1.956 & 1.9503 & 1.9500 & 1.9564 \\
0.5 & 2.111 & 2.117 & 2.128 & 2.1147 & 2.1144 & 2.1283 \\
0.55 & 2.294 & 2.302 & 2.326 & 2.2986 & 2.2981 & 2.3256 \\
0.6 & 2.500 & 2.511 & 2.556 & 2.5055 & 2.5049 & 2.5561 \\
0.65 & 2.733 & 2.750 & 2.832 & 2.7401 & 2.7394 & 2.8319 \\
0.7 & 3.000 & 3.024 & 3.173 & 3.0083 & 3.0075 & 3.1731 \\
0.75 & 3.308 & 3.344 & 3.620 & 3.3180 & 3.3170 & 3.6197 \\
\hline
\end{tabular}

Results are given in GPa.

Table 9.6 Comparison of the effective coefficient $C_{1313}^{*}$ normalized by the shear modulus of matrix $\mu_{0}$ with Table 8 of Mogilevskaya et al (2014), $\gamma=\Gamma=0.001$ for fibre inclusions in the composite matrix/high inclusion

\begin{tabular}{lllllll}
\hline$v_{f}$ & Eq. (6) & Eq. (20) & RUC & PM-DDF1 & PM-DDF2 & SAFEM \\
\hline 0.05 & 1.105 & 1.106 & 1.105 & 1.1239 & 1.1220 & 1.1047 \\
0.1 & 1.221 & 1.224 & 1.221 & 1.2641 & 1.2598 & 1.2209 \\
0.15 & 1.351 & 1.355 & 1.351 & 1.4241 & 1.4168 & 1.3509 \\
0.2 & 1.497 & 1.502 & 1.497 & 1.6085 & 1.5972 & 1.4972 \\
0.25 & 1.662 & 1.668 & 1.663 & 1.8232 & 1.8067 & 1.6633 \\
0.3 & 1.851 & 1.858 & 1.854 & 2.0765 & 2.0529 & 1.8540 \\
0.35 & 2.069 & 2.077 & 2.076 & 2.3797 & 2.3466 & 2.0762 \\
0.4 & 2.322 & 2.333 & 2.340 & 2.7492 & 2.7027 & 2.3396 \\
0.45 & 2.622 & 2.635 & 2.659 & 3.2094 & 3.1437 & 2.6590 \\
0.5 & 2.980 & 2.999 & 3.058 & 3.7983 & 3.7039 & 3.0583 \\
0.55 & 3.418 & 3.446 & 3.578 & 4.5789 & 4.4392 & 3.5779 \\
0.6 & 3.963 & 4.008 & 4.294 & 5.6628 & 5.4471 & 4.2939 \\
0.65 & 4.662 & 4.737 & 5.372 & 7.2694 & 6.9133 & 5.3719 \\
0.7 & 5.590 & 5.720 & 7.274 & 9.8970 & 9.2429 & 7.2737 \\
0.75 & 6.882 & 7.123 & 12.227 & 14.9721 & 13.5163 & 12.2267 \\
\hline
\end{tabular}

The results are given in GPa.

normalized by the matrix coefficient $C_{1133}^{m}$ are shown in Tables 9.7 and 9.8, respectively. Comparisons are given by present model, Mogilevskaya et al (2014), SAFEM reported in Otero et al $(2013,2017)$ and asymtotic homogenization method (AHM) proposed in Bravo-Castillero et al (2012); Guinovart-Diaz et al (2001). Aspect ratio parameters 
Table 9.7 Comparison of the effective coefficient $\left(C_{1111}^{*}-C_{1122}^{*}\right) / 2$ normalized by the shear modulus of matrix $\mu_{0}$ with Table 4 of Mogilevskaya et al (2014), $\gamma=\Gamma=0.001$ for fibre inclusions in the composite matrix/moderate inclusion

\begin{tabular}{lllllllll}
\hline$v_{f}$ & Eq. (4) & Eq. (13) & Eq. (18) & RUC & PM-DDF1 & PM-DDF2 & SAFEM & AHM \\
\hline 0.05 & 1.066 & 1.069 & 1.069 & 1.069 & 1.063 & 1.063 & 1.0653 & 1.0653 \\
0.1 & 1.137 & 1.148 & 1.150 & 1.148 & 1.1312 & 1.1312 & 1.1409 & 1.1409 \\
0.15 & 1.214 & 1.242 & 1.244 & 1.241 & 1.2052 & 1.2052 & 1.2281 & 1.2281 \\
0.2 & 1.298 & 1.349 & 1.352 & 1.349 & 1.2858 & 1.2857 & 1.3285 & 1.3285 \\
0.25 & 1.389 & 1.474 & 1.477 & 1.474 & 1.3739 & 1.3739 & 1.4438 & 1.4438 \\
0.3 & 1.489 & 1.616 & 1.621 & 1.617 & 1.4707 & 1.4706 & 1.5755 & 1.5755 \\
0.35 & 1.600 & 1.777 & 1.783 & 1.780 & 1.5774 & 1.5774 & 1.7257 & 1.7257 \\
0.4 & 1.722 & 1.958 & 1.965 & 1.965 & 1.6958 & 1.6958 & 1.8963 & 1.8963 \\
0.45 & 1.858 & 2.157 & 2.166 & 2.172 & 1.8277 & 1.8277 & 2.0894 & 2.0893 \\
0.5 & 2.010 & 2.374 & 2.385 & 2.403 & 1.9758 & 1.9758 & 2.3070 & 2.3069 \\
0.55 & 2.181 & 2.604 & 2.616 & 2.656 & 2.1431 & 2.143 & 2.5516 & 2.5512 \\
0.6 & 2.376 & 2.841 & 2.856 & 2.929 & 2.3336 & 2.3336 & 2.8253 & 2.8243 \\
0.65 & 2.599 & 3.078 & 3.096 & 3.218 & 2.5526 & 2.5525 & 3.1309 & 3.1282 \\
0.7 & 2.857 & 3.306 & 3.327 & 3.517 & 2.8069 & 2.8068 & 3.4718 & 3.4650 \\
0.75 & 3.158 & 3.514 & 3.538 & 3.823 & 3.1058 & 3.1057 & 3.8532 & 3.8369 \\
\hline
\end{tabular}

Results are given in GPa.

Table 9.8 Comparison of the effective coefficient $C_{1133}^{*}$ normalized by the coefficient $C_{1133}^{m}$ of the matrix with Table 9 of Mogilevskaya et al (2014), $\gamma=\Gamma=0.001$ for fibre inclusions in the composite matrix/high inclusion

\begin{tabular}{llllllll}
\hline$v_{f}$ & Eq. (7) & Eq. (21) & RUC & PM-DDF1 & PM-DDF2 & SAFEM & AHM \\
\hline 0.05 & 1.032 & 1.033 & 1.032 & 1.1045 & 1.1036 & 1.0956 & 1.0956 \\
0.1 & 1.068 & 1.069 & 1.068 & 1.2206 & 1.2188 & 1.2019 & 1.2019 \\
0.15 & 1.109 & 1.11 & 1.109 & 1.3505 & 1.3475 & 1.3206 & 1.3206 \\
0.2 & 1.154 & 1.156 & 1.154 & 1.4965 & 1.4923 & 1.4542 & 1.4542 \\
0.25 & 1.205 & 1.208 & 1.205 & 1.662 & 1.6564 & 1.6058 & 1.6058 \\
0.3 & 1.263 & 1.267 & 1.264 & 1.8512 & 1.844 & 1.7796 & 1.7796 \\
0.35 & 1.33 & 1.335 & 1.331 & 2.0695 & 2.0605 & 1.9810 & 1.9810 \\
0.4 & 1.409 & 1.415 & 1.411 & 2.3242 & 2.313 & 2.2184 & 2.2184 \\
0.45 & 1.501 & 1.509 & 1.506 & 2.6252 & 2.6114 & 2.5038 & 2.5037 \\
0.5 & 1.611 & 1.621 & 1.623 & 2.9863 & 2.9694 & 2.8569 & 2.8564 \\
0.55 & 1.746 & 1.757 & 1.772 & 3.4276 & 3.4069 & 3.3119 & 3.3101 \\
0.6 & 1.913 & 1.927 & 1.974 & 3.9792 & 3.9537 & 3.9348 & 3.9278 \\
0.65 & 2.127 & 2.142 & 2.275 & 4.6881 & 4.6564 & 4.8737 & 4.8447 \\
0.7 & 2.411 & 2.427 & 2.804 & 5.6329 & 5.593 & 6.5509 & 6.4123 \\
0.75 & 2.804 & 2.819 & 4.177 & 6.9548 & 6.9033 & 11.0435 & 9.9304 \\
\hline
\end{tabular}

Results are given in GPa.

$\gamma$ and $\Gamma$ in the calculations are taken the same as in Tables 9.5 and 9.6, $\lambda=100$ and $s=3700$.

A validation of the present model (PM) with experimental data reported by Premkumar et al (1992); Liaw et al (1995); El-Eskandarany (1998); Kumar et al 
(2009); Upadhyay and Singh (2012); Qu et al (2016) is shown in Fig. 9.5. The experimental and theoretical studies are for the effective Young modulus for different volume fraction of $\mathrm{SiC}$ isotropic spherical inclusions into Aluminum isotropic matrix, where the properties are given in Table 9.1. The experimental data is reported for different percentages of volume fraction. PM predictions is consistent with Liaw et al (1995); Qu et al (2016); Upadhyay and Singh (2012)

$$
E_{t}^{*}=\frac{1}{S_{1111}^{*}}, \quad E_{a}^{*}=\frac{1}{S_{3333}^{*}}, \quad \mathbf{S}^{*}=\left(\mathbf{C}^{*}\right)^{-1}
$$

For isotropic composites the effective axial $E_{a}^{*}$ and tangential $E_{t}^{*}$ Young modulus are the same and are given by Eq. (9.26) taking into account that $S_{1111}^{*}=S_{3333}^{*}$.

\subsubsection{Study of Composites Constituted by Isotropic Matrix and Transversely Isotropic Inhomogeneities}

Figure 9.6 displays another validation of the present model approach for the effective axial shear modulus of a material composed by isotropic matrix 3501-6 Epoxy and AS4 Graphite with transversely isotropic symmetry inclusions. The inclusions are embedded into the matrix. The elastic properties of the constituents are given in Table 9.1. This validation assures good concordance with numerical predictions

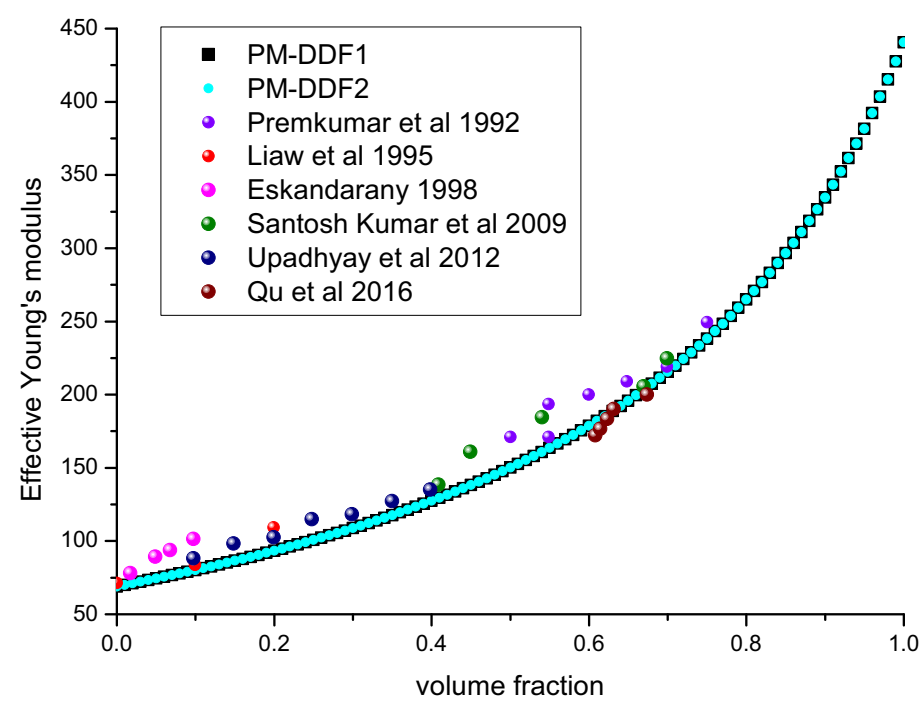

Fig. 9.5 Effective Young's modulus prediction between PM and experimental data reported in Premkumar et al (1992); Liaw et al (1995); El-Eskandarany (1998); Kumar et al (2009); Upadhyay and Singh (2012); Qu et al (2016), $\gamma=\Gamma=1$ for spherical inclusions 


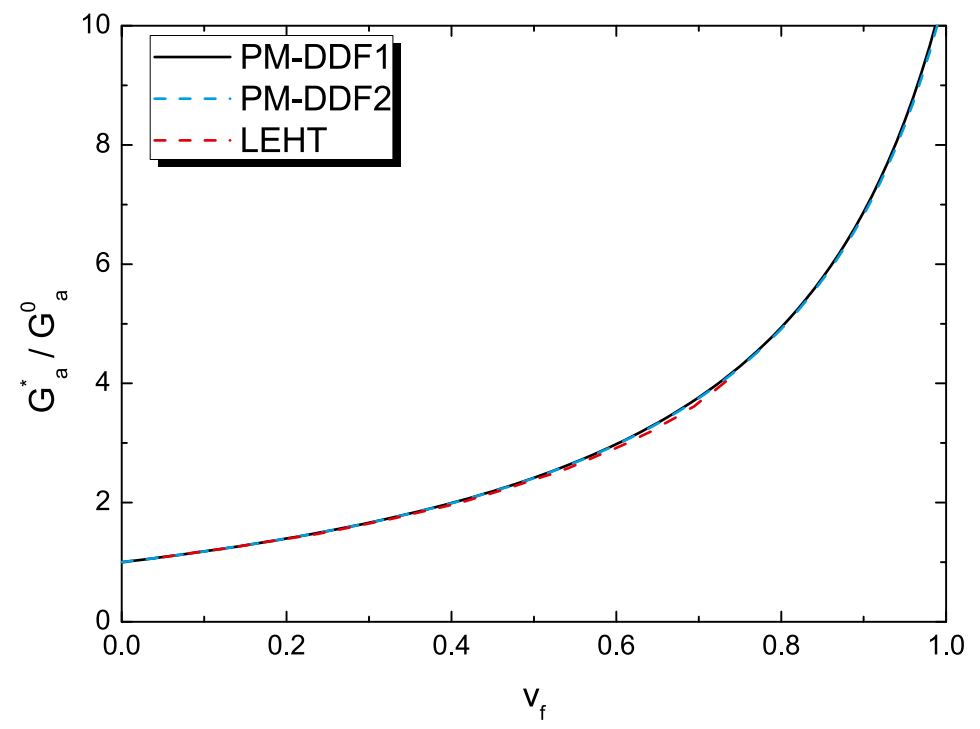

Fig. 9.6 Comparison between PM and LEHT (Wang and Pindera, 2016) approaches for the effective axial shear modulus in a composite made of isotropic matrix 3501-6 Epoxy and AS4 Graphite with transversely isotropic symmetry, $\gamma=\Gamma=0.01$ for fibre inclusions

reported by LEHT homogenization approach (Wang and Pindera, 2016). In Fig. 9.6 are shown numerical predictions for effective axial shear modulus of composite 3501-6 Epoxy/AS4 Graphite (Table 9.1) related to the dependence of the volume fraction of fibre inclusions. A comparison between PM using both density distribution functions (DDF) and Locally Exact Homogenization Theory (LEHT) is done. In both models PM-DDF1 and PM-DDF2, the aspect ratio of fictitious building and fibre inclusions are $\gamma=\Gamma=0.01$, disorder parameters in DDF1 and DDF2 are $\lambda=100$ and $s=3700$, respectively for modeling the case of aligned fibres inside the matrix material. From Fig. 9.6, PM and LEHT give very close results for this effective property of the composite in the whole range of volume fraction.

\subsubsection{Porous Composite with Isotropic and Transversely Isotropic Matrix}

The next study focus on numerical prediction of porous composites. Validation of PM with Maxwell approach reported by Sevostianov (2014) (as SMM), Vilchevskaya and Sevostianov (2015) (as Max. schem.) and experimental results reported in Dong and Guo (2004) is shown in Fig. 9.7. The effective transversal shear modulus of two phase composite matrix2/porous (see Table 9.1) is compared between PM, Max. schem. 

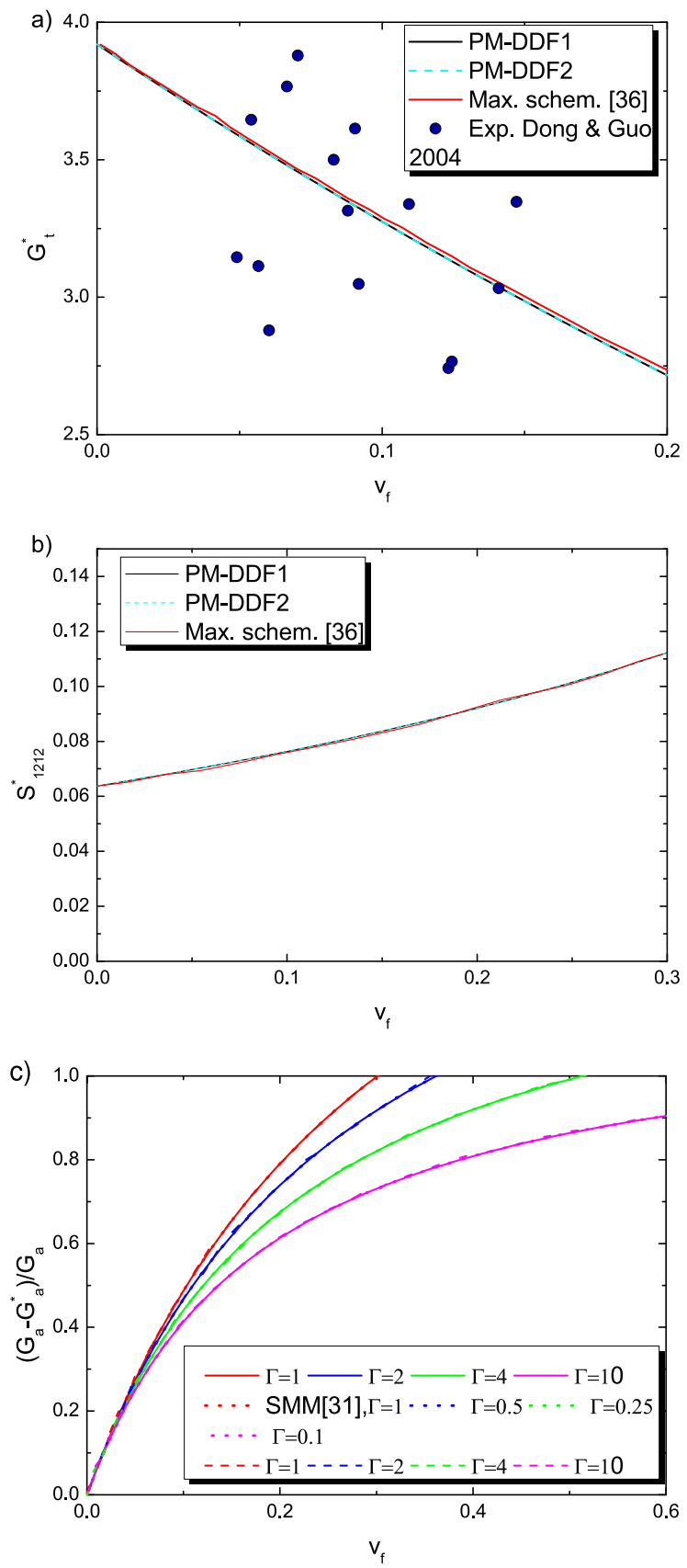

Fig. 9.7 Effective coefficient a) $G_{t}^{*}$, b) $S_{1212}^{*}$ for matrix $2 /$ porous composite, $\gamma=\Gamma=1 / 1.39$ for fibre spheroidal porous inclusions, and c) $G_{a}-G_{a}^{*}$ for matrix 1/porous composite normalized by axial shear modulus $G_{a}$ of matrix material, $\gamma=10$ for disk spheroidal porous inclusions at four fixed values of $\Gamma$ 
Vilchevskaya and Sevostianov (2015) and the experimental data reported by Dong and Guo (2004) in Fig. 9.7a). The matrix2 exhibits transversely isotropic symmetry. In both approaches of PM, i.e. PM-DDF1 and PM-DDF2, are taken $\gamma=\Gamma=1 / 1.39$, $\lambda=100$ for PM-DDF1 and $s=3700$ for PM-DDF2 as disorder parameters for modeling the porous inclusion parallel aligned case. From Fig. 9.7a) one can observe that PM-DDF1 and PM-DDF2 approaches give close results, while both approach of the present model (PM-DDF1 and PM-DDF2) have good correspondence with Max. schem. Vilchevskaya and Sevostianov (2015) approach and experimental data of Dong and Guo (2004). In Fig. 9.7b) it is shown a numerical comparison for the effective compliance coefficient $S_{1212}^{*}$ between PM and Max. schem. reported in Vilchevskaya and Sevostianov (2015), for two phase composite matrix2/porous of Table 9.1. In both predictions of PM approach (PM-DDF1 and PM-DDF2), are taken the same values of $\gamma, \Gamma, \lambda$ and $s$ like in Fig.9.7a). From Fig. 9.7b) one can observe that PM-DDF1, PM-DDF2 and Max. schem. Vilchevskaya and Sevostianov (2015) approaches practically coincide in the whole range of volume fraction. In Fig. 9.7c) are shown the numerical comparison of the effective axial coefficient $G_{a}-G_{a}^{*}$ normalized by the matrix material axial shear modulus $G_{a}$ for two phase composite constituted by matrix 1/porous of Table 9.1 between PM and SMM reported in Sevostianov (2014). The solid and dash curves indicate PM-DDF1 and PM-DDF2 approaches, respectively. The constituent matrix 1 exhibit isotropic symmetry. The porous aspect ratio are taken as $\gamma=10$ and the aspect ratio of fictitious building $\Gamma$ is fixed as $\Gamma=1,2,4,10$ to see the influence of different geometrical shape of fictitious building on the behavior of this effective coefficient. From Fig. 9.7c), it is observed that when the effective coefficient $\left(G_{a}-G_{a}^{*}\right) / G_{a}>1$ then $G_{a}^{*}<0$, therefore, this fact conduces to lost of physical meaning, thus the only curve with real physical meaning is that none having value $\Gamma=10$, i.e., the same aspect ratio for inclusions and fictitious building. For PM-DDF1 is taken $\lambda=100$ and for PM-DDF2 $s=3700$. Comparisons between the two approaches of PM (PM-DDF1 and PM-DDF2) and numerical results (SMM) shown in Fig. 9.7b) of Sevostianov (2014) give close results. Furthermore, in Table 9.9, numerical comparisons are reported using the present model and SAFEM for the effective transversal shear modulus $G_{t}^{*}$ in the composite matrix $2 /$ porous. There is a good match between results obtained through both approaches.

Table 9.9 Comparison between the present model and SAFEM approach for the effective transversal $G_{t}^{*}$ shear modulus in the composite made by fibre spheroidal porous inhomogeneities embedded into the matrix $2, \gamma=\Gamma=1 / 1.39, \lambda=100, s=3700$

\begin{tabular}{|c|c|c|c|}
\hline$v_{f}$ & SAFEM $G_{t}^{*} \quad(\mathrm{GPa})$ & PM-DDF1 $G_{t}^{*} \quad(\mathrm{GPa})$ & $\mathrm{PM}-\mathrm{DDF} 2 G_{t}^{*} \quad(\mathrm{GPa})$ \\
\hline 0.1 & 3.3354 & 3.2749 & 3.275 \\
\hline 0.2 & 2.7686 & 2.7161 & 2.7162 \\
\hline 0.3 & 2.2516 & 2.2273 & 2.2274 \\
\hline
\end{tabular}




\subsubsection{Two-phase Nano-composites}

The last validation of the present model (PM) is done for the case of a two phase nano-composite constituted by isotropic matrix LaRC-SI and continuum graphene as inclusions with isotropic symmetry. The properties of these materials are shown in Table 9.1. In Fig. 9.8 are shown numerical predictions of PM-DDF1 and PM-DDF2 approaches for the effective axial $G_{a}^{*}$ and transversal $G_{t}^{*}$ shear modulus normalized by the corresponding axial $G_{a}$ and transversal $G_{t}$ shear modulus of matrix material in a two phase nanocomposite LaRC-SI/continuum graphene. The effective axial $G_{a}^{*}$ and transversal $G_{t}^{*}$ shear modulus calculated by PM are validated with three different methods (the Sequential, the Two-level based on Mori-Tanaka method and the FE approaches) reported in Selmi et al (2007) for nanocomposites. The parameters for PM predictions are taken $\gamma=\Gamma=0.1, \lambda=100$ and $s=3700$ for PM-DDF1 and PM-DDF2, respectively. Figure 9.8 evidences that PM-DDF1 and PM-DDF2 match very good with Sequential, Two-level and FE approaches reported in Selmi et al (2007).

\subsection{Conclusions}

From the research that has been carried out, it is possible to conclude that based on the results obtained, Maxwell homogenization approach has been very successful as a procedure to estimate effective elastic properties of composites constituted by phases of transversely isotropic symmetry. A methodology for computing the overall properties is reported from the outcome of our investigation. Closed formulas for effective stiffness tensor of composites with different arrangement of the inclusions have been deduced. Current homogenization technique is compared with other theoretical approaches, getting low computational cost of the implemented algorithm
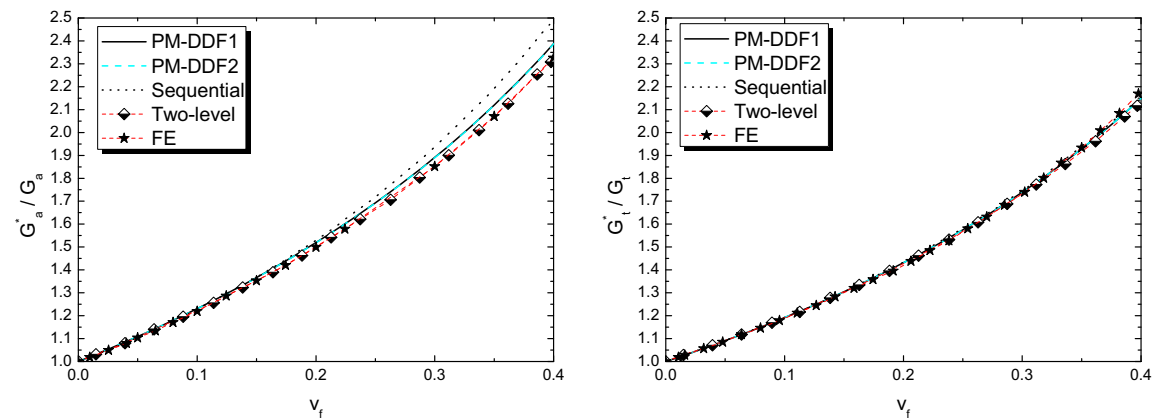

Fig. 9.8 Effective axial $G_{a}^{*}$ and transversal $G_{t}^{*}$ shear modulus normalized by the matrix material axial $G_{a}$ and transversal $G_{t}$ shear modulus, respectively, for a composite LaRC-SI/continuum Graphene, $\gamma=\Gamma=0.1$ for fibre inclusions 
derived from the present model and correlating satisfactorily with the results reported in the studied references. The used method is especially reachable and forthright procedure for the calculation of effective properties of heterogeneous media with more of two different types of constituents. As stated in the introduction our main target was to hand over new analytical formulae for the control alignment tensors $\mathbf{N}^{*}$ and $\mathbf{N}^{s *}$ which are emphasized in the appendix of the current contribution. Besides, an study about Maxwell approach using two different density distribution functions is done for predicting effective mechanical properties of transversely isotropic, nano and porous composites.

\section{Appendix}

Tensor $\mathbf{P}$ of Maxwell explicit effective formula (9.24) for transversely isotropic spheroidal inclusions of aspect ratio $\gamma=\frac{x_{1}}{x_{3}}$, where $x_{1}, x_{2}, x_{3}$ are the inclusion's axis, is given by

$$
\begin{aligned}
\mathbf{P}_{1111}= & \frac{1}{16}\left(\int_{-1}^{1} \frac{\left(1-u^{2}\right) \cdot \Upsilon(u)}{b} d u\right)+\frac{3}{16}\left(\int_{-1}^{1} \frac{d\left(1-u^{2}\right) \cdot \Upsilon(u)}{h_{0}+h_{1} u^{2}+h_{2} u^{4}} d u\right), \\
\mathbf{P}_{1122}= & -\frac{1}{16}\left(\int_{-1}^{1} \frac{\left(1-u^{2}\right) \cdot \Upsilon(u)}{b} d u\right)+\frac{1}{16}\left(\int_{-1}^{1} \frac{d\left(1-u^{2}\right) \cdot \Upsilon(u)}{h_{0}+h_{1} u^{2}+h_{2} u^{4}} d u\right), \\
\mathbf{P}_{1133}= & -\frac{\left(C_{1133}+C_{2323}\right)}{4}\left(\int_{-1}^{1} \frac{u^{2} \cdot \Upsilon(u)}{h_{0}+h_{1} u^{2}+h_{2} u^{4}} d u\right)+ \\
& +\frac{\left(C_{1133}+C_{2323}\right)}{4}\left(\int_{-1}^{1} \frac{u^{4} \cdot \Upsilon(u)}{h_{0}+h_{1} u^{2}+h_{2} u^{4}} d u\right), \\
\mathbf{P}_{3333}= & \frac{C_{1111}}{2}\left(\int_{-1}^{1} \frac{u^{2} \cdot \Upsilon(u)}{h_{0}+h_{1} u^{2}+h_{2} u^{4}} d u\right)+ \\
& +\frac{\left(C_{2323}-C_{1111}\right)}{2}\left(\int_{-1}^{1} \frac{u^{4} \cdot \Upsilon(u)}{h_{0}+h_{1} u^{2}+h_{2} u^{4}} d u\right), \\
\mathbf{P}_{2323}= & -\frac{\left(C_{1133}+C_{1111}\right)}{8} \cdot\left(\int_{-1}^{1} \frac{u^{2} \cdot \Upsilon(u) d u}{h_{0}+h_{1} u^{2}+h_{2} u^{4}}\right)+\frac{1}{16} \cdot\left(\int_{-1}^{1} \frac{u^{2} \cdot \Upsilon(u) d u}{b}\right)+ \\
& +\frac{C_{1111}}{16} \cdot\left(\int_{-1}^{1} \frac{\Upsilon(u) d u}{h_{0}+h_{1} u^{2}+h_{2} u^{4}}\right)+ \\
& +\frac{\left[2 C_{1133}+C_{3333}+C_{1111}\right]}{16} \cdot\left(\int_{-1}^{1} \frac{u^{4} \cdot \Upsilon(u) d u}{h_{0}+h_{1} u^{2}+h_{2} u^{4}}\right),
\end{aligned}
$$




$$
\begin{aligned}
\mathbf{P}_{1212}= & \frac{\mathbf{P}_{1111}-\mathbf{P}_{1122}}{2}=\frac{1}{16} \cdot\left(\int_{-1}^{1} \frac{\left(1-u^{2}\right) \cdot \Upsilon(u)}{b} d u\right)+ \\
& +\frac{1}{16} \cdot\left(\int_{-1}^{1} \frac{d\left(1-u^{2}\right) \cdot \Upsilon(u)}{\left(h_{0}+h_{1} u^{2}+h_{2} u^{4}\right)} d u\right)
\end{aligned}
$$

where

$$
\Upsilon(u)=\frac{\gamma^{2}}{\left[\gamma^{2}+u^{2}\left(1-\gamma^{2}\right)\right]^{3 / 2}},
$$

and

$$
\begin{aligned}
b & =\frac{\left(C_{1111}-C_{1122}\right)}{2}\left(1-u^{2}\right)+C_{2323} u^{2}, \quad d=C_{2323}\left(1-u^{2}\right)+C_{3333} u^{2}, \\
h_{0} & =C_{1111} C_{2323}, \quad h_{1}=C_{1111} C_{3333}-C_{1133}^{2}-2 C_{1133} C_{2323}-2 C_{1111} C_{2323}, \\
h_{2} & =C_{3333} C_{2323}+C_{1111} C_{2323}+2 C_{1133} C_{2323}+C_{1133}^{2}-C_{1111} C_{3333} .
\end{aligned}
$$

Using decomposition (9.16), (9.17) and eq. (9.27), it holds

$$
\mathbf{P}=\left(p_{1}, p_{2}, p_{3}, p_{4}, p_{5}, p_{6}\right),
$$

where

$$
\begin{aligned}
& p_{1}=\frac{\mathbf{P}_{1111}+\mathbf{P}_{1122}}{2}, p_{2}=\mathbf{P}_{1111}-\mathbf{P}_{1122}, p_{3}=p_{4}=\mathbf{P}_{1133}, \\
& p_{5}=4 \mathbf{P}_{2323}, p_{6}=\mathbf{P}_{3333} .
\end{aligned}
$$

Tensor $\mathbf{N}$ of Maxwell explicit effective formula (9.24) for transversely isotropic spheroidal inclusions, is given by the relation

$$
\mathbf{N}=\left[\left(\mathbf{C}^{1}-\mathbf{C}^{0}\right)^{-1}+\mathbf{P}\right]^{-1}
$$

where $\mathbf{C}^{0}$ and $\mathbf{C}^{1}$ are the stiffness tensor of matrix and inclusions material, respectively. Explicitly

$$
\mathbf{N}=\left(n_{1}, n_{2}, n_{3}, n_{4}, n_{5}, n_{6}\right),
$$

where

$$
\begin{aligned}
& n_{1}=\frac{1}{2}\left[\frac{d_{1}+d_{2}}{d_{5}\left(d_{1}+d_{2}\right)-2 d_{3}^{2}}+p_{6}\right], \quad n_{2}=\frac{d_{1}-d_{2}}{1+p_{2}\left(d_{1}-d_{2}\right)}, \\
& n_{3}=n_{4}=-\frac{1}{\bullet}\left[p_{3}-\frac{d_{3}}{d_{5}\left(d_{1}+d_{2}\right)-2 d_{3}^{2}}\right], \quad n_{5}=\frac{4 d_{4}}{1+p_{5} d_{4}},
\end{aligned}
$$




$$
\begin{aligned}
n_{6} & =\frac{1}{\diamond}\left[\frac{d_{5}}{d_{5}\left(d_{1}+d_{2}\right)-2 d_{3}^{2}}+2 p_{1}\right], \\
\nabla & =\frac{1+p_{6} d_{5}+2 p_{1}\left(d_{1}+d_{2}\right)+4 p_{3} d_{3}}{d_{5}\left(d_{1}+d_{2}\right)-2 d_{3}^{2}}+2 p_{1} p_{6}-2 p_{3}^{2}, \\
& d_{1}=C_{1111}^{1}-C_{1111}^{0}, d_{2}=C_{1122}^{1}-C_{1122}^{0}, d_{3}=C_{1133}^{1}-C_{1133}^{0}, \\
d_{4} & =C_{2323}^{1}-C_{2323}^{0}, d_{5}=C_{3333}^{1}-C_{3333}^{0},
\end{aligned}
$$

where $p_{i}, i=1,2, \cdots, 6$ are given by eq. (9.33). Remark that this explicit expressions for components $n_{i}, i=1,2, \ldots, 6$ used in the decomposition of tensor $\mathbf{N}$ into basis $\mathbf{T}$ given by (9.12)-(9.14) are different and more general than explicit expressions given by formulae (A.9) and (A.10) of Sevostianov (2014), because they are for a material with transversely isotropic symmetry.

Expression of tensor $\mathbf{Q}$ for transversely isotropic spheroidal inclusions, is given by the relation

$$
\mathbf{Q}=\mathbf{C}^{0} \cdot\left(\mathbf{I}-\mathbf{P} \cdot \mathbf{C}^{0}\right)
$$

where $\mathbf{C}^{0}$ is the stiffness tensor of matrix material. Explicitly

$$
\mathbf{Q}=\left(q_{1}, q_{2}, q_{3}, q_{4}, q_{5}, q_{6}\right)
$$

and

$$
\begin{gathered}
q_{1}=\frac{1}{2} D_{a}\left(1-2 p_{1} D_{a}-4 p_{3} C_{13}^{0}\right)-p_{6} D_{c}, \quad q_{2}=D_{b}\left(1-p_{2} D_{b}\right), \\
q_{3}=q_{4}=C_{13}^{0} W_{a}-D_{a} W_{b}-2 p_{3} D_{c}, \quad q_{5}=4 C_{44}^{0} W_{c}, \\
q_{6}=C_{33}^{0} W_{a}-4 C_{13}^{0}\left(W_{b}-p_{1} C_{13}^{0}\right), \\
D_{a}=C_{11}^{0}+C_{12}^{0}, \quad D_{b}=C_{11}^{0}-C_{12}^{0}, \quad D_{c}=\left(C_{13}^{0}\right)^{2}, \\
W_{a}=1-p_{6} C_{33}^{0}, \quad W_{b}=2 p_{1} C_{13}^{0}+p_{3} C_{33}^{0}, \quad W_{c}=1-p_{5} C_{44}^{0},
\end{gathered}
$$

where $p_{i}, i=1, \ldots 6$, are the components of tensor $\mathbf{P}$ of eq. (9.33). Remark that this explicit expressions for components $q_{i}, i=1,2, \ldots, 6$ used in the decomposition of tensor $\mathbf{Q}$ into basis $\mathbf{T}$ given by (9.12)-(9.14) are different and more general than explicit expressions given by formulae (A.6) of Sevostianov (2014), because they are for a material with transversely isotropic symmetry.

Then, introducing parameter $\lambda$ Sevostianov (2014) that controls the random or not random aligned inclusions cases, by means of density distribution function

$$
P_{\lambda}(\psi)=\frac{1}{2 \pi}\left[\left(1+\lambda^{2}\right) e^{-\lambda \psi}+\lambda e^{-\lambda \frac{\pi}{2}}\right]
$$

using eq. (9.36)-(9.38), thus, tensor $\mathbf{N}^{*}$ can be calculated by 


$$
\mathbf{N}_{i j k l}^{*}=\int_{0}^{\frac{\pi}{2}} P_{\lambda}(\psi) \sin \psi d \psi \int_{0}^{2 \pi}\left(\sum_{p=1}^{6} n_{p} \mathbf{T}_{i j k l}^{p}\right) d \theta
$$

Explicitly,

$$
\begin{aligned}
& N_{1111}^{*}=\frac{1}{2}\left[2\left(w_{1}+w_{2}\right)+2\left(w_{3}+w_{4}\right) g_{1}(\lambda)+\frac{3}{4} w_{5} g_{2}(\lambda)\right], \\
& N_{1122}^{*}=\frac{1}{2}\left[2 w_{1}+2 w_{3} g_{1}(\lambda)+\frac{1}{4} w_{5} g_{2}(\lambda)\right], \\
& N_{3333}^{*}=\frac{1}{2}\left[2\left(w_{1}+w_{2}\right)+2\left(w_{3}+w_{4}\right) g_{3}(\lambda)+2 w_{5} g_{4}(\lambda)\right], \\
& N_{1133}^{*}=\frac{1}{2}\left[2 w_{1}+2 w_{3} g_{5}(\lambda)+2 w_{5} g_{6}(\lambda)\right], \\
& N_{1313}^{*}=\frac{1}{2}\left[w_{2}+w_{4} g_{5}(\lambda)+2 w_{5} g_{6}(\lambda)\right] \\
& N_{1212}^{*}=\frac{N_{1111}^{*}-N_{1122}^{*}}{2},
\end{aligned}
$$

with

$$
\begin{aligned}
& w_{1}=n_{1}-\frac{n_{2}}{2}, \quad w_{2}=n_{2}, \quad w_{3}=2 n_{3}+n_{2}-2 n_{1}, \\
& w_{4}=n_{5}-2 n_{2}, \quad w_{5}=n_{6}+n_{1}+\frac{n_{2}}{2}-2 n_{3}-n_{5},
\end{aligned}
$$

and functions $g_{j}(\lambda), j=1,2, \cdots, 6$ are given by formulas

$$
\begin{aligned}
& g_{1}(\lambda)=\frac{18-\lambda\left(\lambda^{2}+3\right) e^{-\frac{\lambda \pi}{2}}}{6\left(\lambda^{2}+9\right)}, \\
& g_{2}(\lambda)=\frac{120}{\left(\lambda^{2}+9\right)\left(\lambda^{2}+25\right)}-\lambda \frac{7 \lambda^{4}+178 \lambda^{2}+435}{15\left(\lambda^{2}+9\right)\left(\lambda^{2}+25\right)} e^{-\frac{\lambda \pi}{2}}, \\
& g_{3}(\lambda)=\frac{\left(\lambda^{2}+3\right)\left(3+\lambda e^{-\lambda \pi / 2}\right)}{3\left(\lambda^{2}+9\right)}, \\
& g_{4}(\lambda)=\frac{24+\left(\lambda^{2}+1\right)\left(\lambda^{2}+21\right)}{\left(\lambda^{2}+9\right)\left(\lambda^{2}+25\right)}+\lambda e^{-\lambda \pi / 2} \frac{\left[\left(\lambda^{2}+9\right)\left(\lambda^{2}+25\right)-120\right]}{5\left(\lambda^{2}+9\right)\left(\lambda^{2}+25\right)}, \\
& g_{5}(\lambda)=\frac{3}{2\left(\lambda^{2}+9\right)}+\frac{\left(\lambda^{2}+3\right)\left(6+\lambda e^{-\lambda \pi / 2}\right)}{12\left(\lambda^{2}+9\right)}, \\
& g_{6}(\lambda)=\frac{3\left(\lambda^{2}+5\right)}{\left(\lambda^{2}+9\right)\left(\lambda^{2}+25\right)}+\frac{\lambda\left[\left(\lambda^{2}+1\right)\left(\lambda^{2}+18\right)+12\right]}{15\left(\lambda^{2}+9\right)\left(\lambda^{2}+25\right)} e^{-\lambda \pi / 2} .
\end{aligned}
$$


Remark that explicitly expressions obtained here for functions $g_{j}(\lambda), j=1,2, \ldots, 6$ are different of explicit expressions obtained for functions $g_{i}(\lambda)$ reported in formula (3.6) of Sevostianov (2014), except for functions $g_{1}(\lambda), g_{4}(\lambda)$ that coincides in both contributions.

On the other hand, one can replace density distribution function given by (9.47) by density distribution function

$$
W_{s}(\psi)=\frac{1}{4 \pi} \frac{s \cdot \cosh (s \cdot \cos \psi)}{\sinh s},
$$

in which the parameter $s$ is the disorder parameter, and similar to Eq. (9.48), one can calculate tensor $\mathbf{N}^{s *}$ given by

$$
\mathbf{N}_{i j k l}^{s *}=\int_{0}^{\pi} W_{s}(\psi) \sin \psi d \psi \int_{0}^{2 \pi}\left(\sum_{p=1}^{6} n_{p} T_{i j k l}^{p}\right) d \theta,
$$

which explicitly is written

$$
\begin{aligned}
& \mathbf{N}_{1111}^{s *}=\frac{1}{4}\left[4\left(w_{1}+w_{2}\right)+4\left(w_{3}+w_{4}\right) \tau_{1}(s)+\frac{3}{4} w_{5} \tau_{2}(s)\right] \\
& \mathbf{N}_{1122}^{s *}=\frac{1}{4}\left[4 w_{1}+4 w_{3} \tau_{1}(s)+\frac{1}{4} w_{5} \tau_{2}(s)\right] \\
& \mathbf{N}_{3333}^{s *}=\frac{1}{4}\left[4\left(w_{1}+w_{2}\right)+4\left(w_{3}+w_{4}\right) \tau_{3}(s)+2 w_{5} \tau_{4}(s)\right] \\
& \mathbf{N}_{1133}^{s *}=\frac{1}{4}\left[4 w_{1}+\frac{w_{3}}{2} \tau_{5}(s)+4 w_{5} \tau_{6}(s)\right] \\
& \mathbf{N}_{1313}^{s *}=\frac{1}{4}\left[2 w_{2}+\frac{w_{4}}{4} \tau_{5}(s)+4 w_{5} \tau_{6}(s)\right] \\
& \mathbf{N}_{1212}^{s *}=\frac{\mathbf{N}_{1111}^{s *}-\mathbf{N}_{1122}^{s *}}{2}
\end{aligned}
$$

where $w_{i}, i=1, \cdots, 5$ are given by Eq. (9.55)-(9.56), and functions $\tau_{i}, i=1, \cdots, 6$ are,

$$
\begin{aligned}
& \tau_{1}(s)=\frac{1}{s}\left[\frac{s-\tanh s}{s \tanh s}\right], \\
& \tau_{2}(s)=\frac{16}{s^{4}}\left[\frac{s^{2} \tanh s-3(s-\tanh s)}{\tanh s}\right], \\
& \tau_{3}(s)=\frac{1}{s^{2}}\left[\frac{s^{2} \tanh s-2(s-\tanh s)}{\tanh s}\right],
\end{aligned}
$$




$$
\begin{aligned}
& \tau_{4}(s)=\frac{2}{s^{4}}\left[\frac{s^{2}\left(s^{2}+8\right) \tanh s-4(s-\tanh s)\left(6+s^{2}\right)}{\tanh s}\right], \\
& \tau_{5}(s)=\frac{4}{s^{2}}\left[\frac{s^{2} \tanh s-(s-\tanh s)}{\tanh s}\right], \\
& \tau_{6}(s)=\frac{1}{s^{4}}\left[\frac{(s-\tanh s)\left(s^{2}+12\right)-4 s^{2} \tanh s}{\tanh s}\right] .
\end{aligned}
$$

Acknowledgements Authors thank to PHC Carlos J. Finlay 2018 project No. 39142TA (FranceCuba), to Proyecto Nacional de Ciencias Básicas titulado Métodos físico-matemáticos para el estudio de nuevos materiales y la propagación de ondas. Aplicaciones, Project No. 7515. The author RR thanks to Departamento de Matemáticas y Mecánica, IIMAS and PREI-DGAPA at UNAM, for the support to his research project and Ramiro Chávez Tovar and Ana Pérez Arteaga for computational assistance.

\section{References}

Bravo-Castillero J, Guinovart-Diaz R, Rodriguez-Ramos R, Sabina FJ, Brenner R (2012) Unified analytical formulae for the effective properties of periodic fibrous composites. Materials Letters 73:68 - 71, doi:10.1016/j.matlet.2011.12.106

Dong XN, Guo XE (2004) The dependence of transversely isotropic elasticity of human femoral cortical bone on porosity. Journal of Biomechanics 37(8):1281-1287, doi:10.1016/j.jbiomech.2003.12.011

Dong XN, Zhang X, Huang YY, Guo XE (2005) A generalized self-consistent estimate for the effective elastic moduli of fiber-reinforced composite materials with multiple transversely isotropic inclusions. International Journal of Mechanical Sciences 47(6):922 - 940, doi:10.1016/j.ijmecsci.2005.01.008

Drago AS, Pindera MJ (2008) A locally-exact homogenization theory for periodic microstructures with isotropic phases. Trans ASME Journal of Applied Mechanics 75(5):051,010-051,010-14, doi:10.1115/1.2913043

El-Eskandarany MS (1998) Mechanical solid state mixing for synthesizing of SiCp/Al nanocomposites. Journal of Alloys and Compounds 279(2):263 - 271, doi:10.1016/S0925-8388(98)00658-6

Giraud A, Huynh Q, Hoxha D, Kondo D (2007) Effective poroelastic properties of transversely isotropic rock-like composites with arbitrarily oriented ellipsoidal inclusions. Mechanics of Materials 39(11):1006 - 1024, doi:10.1016/j.mechmat.2007.05.005

Guinovart-Diaz R, Bravo-Castillero J, Rodriguez-Ramos R, Sabina FJ, Martinez-Rosado R (2001) Overall properties of piezocomposite materials 1-3. Materials Letters 48(2):93 - 98, doi:10.1016/S0167-577X(00)00285-8

Hershey AV (1954) The elasticity of an isotropic aggregate of anisotropic cubic crystals. Trans ASME Journal of Applied Mechanics 21(3):236-240, doi:10.1115/1.2913043

Hill R (1965) A self-consistent mechanics of composite materials. Journal of the Mechanics and Physics of Solids 13(4):213 - 222, doi:10.1016/0022-5096(65)90010-4

Kanaun S (2016) Efficient homogenization techniques for elastic composites: Maxwell scheme vs. effective field method. International Journal of Engineering Science 103:19 - 34, doi:10.1016/j.ijengsci.2016.03.004

Kröner E (1958) Berechnung der elastischen Konstanten des Vielkristalls aus den Konstanten des Einkristalls. Zeischrift für Physik 151(4):504-518, doi:10.1007/BF01337948 
Kumar SS, Bai VS, Rajkumar KV, Sharma GK, Jayakumar T, Rajasekharan T (2009) Elastic modulus of $\mathrm{Al}-\mathrm{Si} / \mathrm{SiC}$ metal matrix composites as a function of volume fraction. Journal of Physics D: Applied Physics 42(17):175,504, doi:10.1088/0022-3727/42/17/175504

Kushch VI, Sevostianov I (2016) Maxwell homogenization scheme as a rigorous method of micromechanics: Application to effective conductivity of a composite with spheroidal particles. International Journal of Engineering Science 98:36 - 50, doi:10.1016/j.ijengsci.2015.07.003, special Issue Dedicated to Sergey Kanaun's 70th Birthday

Levin V, Kanaun S, Markov M (2012) Generalized Maxwell's scheme for homogenization of poroelastic composites. International Journal of Engineering Science 61:75 - 86, doi:10.1016/j.ijengsci.2012.06.011, the special issue in honor of Nikita F. Morozov

Levin VM, Kanaun SK (2012) Application of Maxwell method in solution of homogenization problem for anizotropic elastic media with ellipsoidal inclusions. Proceedings of Petrozavodsk State University 1(8):86-89

Liaw PK, Shannon RE, Clark WG, Harrigan WC, Jeong H, Hsu DK (1995) Nondestructive characterization of material properties of metal-matrix composites. Materials Chemistry and Physics 39(3):220 - 228, doi:10.1016/0254-0584(94)01431-F

Martinez-Ayuso G, Friswell MI, Adhikari S, Khodaparast HH, Berger H (2017) Homogenization of porous piezoelectric materials. International Journal of Solids and Structures 113-114:218 229, doi:10.1016/j.ijsolstr.2017.03.003

Maxwell J (1954) A Treatise on Electricity and Magnetism, 3rd edn. Dover, New York

McCartney LN, Kelly A (2008) Maxwell's far-field methodology applied to the prediction of properties of multi-phase isotropic particulate composites. Proceedings of the Royal Society A: Mathematical, Physical and Engineering Sciences 464(2090):423-446, doi:10.1098/rspa.2007.0071

Mogilevskaya SG, Kushch VI, Nikolskiy D (2014) Evaluation of some approximate estimates for the effective tetragonal elastic moduli of two-phase fiber-reinforced composites. Journal of Composite Materials 48(19):2349-2362, doi:10.1177/0021998313498103

Otero JA, Rodriguez-Ramos R, Bravo-Castillero J, Guinovart-Diaz R, Sabina FJ, Monsivais G (2013) Semi-analytical method for computing effective properties in elastic composite under imperfect contact. International Journal of Solids and Structures 50(3):609 - 622, doi:10.1016/j.ijsolstr.2012.11.001

Otero JA, Rodriguez-Ramos R, Monsivais G (2017) Computation of effective properties in elastic composites under imperfect contact with different inclusion shapes. Mathematical Methods in the Applied Sciences 40(9):3290-3310, doi:10.1002/mma.3956

Premkumar MK, Hunt Jr WH, Sawtell RR (1992) Aluminum composite materials for multichip modules. JOM 44(7):24-28, doi:10.1007/BF03222271

Qu J, Cherkaoui M (2006) Fundamentals of Micromechanics of Solids. John Wiley \& Sons, New Jersey

Qu SG, Lou HS, Li XQ (2016) Influence of particle size distribution on properties of sic particles reinforced aluminum matrix composites with high sic particle content. Journal of Composite Materials 50(8):1049-1058, doi:10.1177/0021998315586864

Roger D, Dieulesaint E (2000) Elastic Waves in Solids, vol I. Springer, New York

Selmi A, Friebel C, Doghri I, Hassis H (2007) Prediction of the elastic properties of single walled carbon nanotube reinforced polymers: A comparative study of several micromechanical models. Composites Science and Technology 67(10):2071 - 2084, doi:10.1016/j.compscitech.2006.11.016

Sevostianov I (2014) On the shape of effective inclusion in the Maxwell homogenization scheme for anisotropic elastic composites. Mechanics of Materials 75:45 - 59, doi:10.1016/j.mechmat.2014.03.003

Sevostianov I, Giraud A (2013) Generalization of Maxwell homogenization scheme for elastic material containing inhomogeneities of diverse shape. International Journal of Engineering Science 64:23 - 36, doi:10.1016/j.ijengsci.2012.12.004

Upadhyay A, Singh R (2012) Prediction of effective elastic modulus of biphasic composite materials. Modern Mechanical Engineering 2(1):6-13, doi:10.4236/mme.2012.21002 
Vilchevskaya E, Sevostianov I (2015) Effective elastic properties of a particulate composite with transversely-isotropic matrix. International Journal of Engineering Science 94:139 - 149, doi:10.1016/j.ijengsci.2015.05.006

Wang G, Pindera MJ (2016) Locally-exact homogenization theory for transversely isotropic unidirectional composites. Mechanics Research Communications 78:2 - 14, doi:10.1016/j.mechrescom.2015.09.011, Recent Advances in Multiscale, Multifunctional and Functionally Graded Materials 